\title{
A New Method for Producing Pharmaceutical Co-crystals: Laser Irradiation of Powder Blends
}

\author{
Varin Titapiwatanakun, Abdul W. Basit, Simon Gaisford* \\ UCL School of Pharmacy, University College London, 29-39 Brunswick Square, \\ London, WC1N 1AX, UK
}

${ }^{*}$ Corresponding author

email: s.gaisford@ucl.ac.uk

Tel: +44(0)20 77535863

Fax: +44(0)20 77535942 


\section{Abstract}

In this work a high-power $\mathrm{CO}_{2}$ laser was used to irradiate powder blends of co-crystal formers, with the specific aim of trying to cause recrystallization to a co-crystal structure. By varying the power and raster speed of the laser, it was found that sufficient thermal energy could be imparted to the powder to cause molecular rearrangement. It was possible to form co-crystals of caffeine with oxalic acid and caffeine with malonic acid. Interestingly, it was found that to form co-crystals successfully, the co-formers needed to sublime to an appreciable extent, which indicates the mechanism of rearrangement involves interaction and nucleation in the vapour phase. Laser irradiation thus offers a new route to creation of pharmaceutical co-crystals and a potentially rapid screen for likely co-crystal formation between coforming pairs.

\section{Key words}

Co-crystal; CO2 laser; caffeine; oxalic acid; malonic acid 


\section{Introduction}

Many pharmaceutical products, especially those designed for oral delivery, contain an active drug in a crystalline state. In cases where the active drug exhibits polymorphism there is frequently a dichotomy between physicochemical properties (dissolution rate, solubility and bioavailability) and stability. Metastable forms usually have better physicochemical properties, but carry the risk of converting to a more stable form during storage. In cases where the bioavailability of a more stable form is unsatisfactory, demonstrating the stability of the metastable form is essential in getting regulatory permission for the product. For instance, the metastable form B of chloramphenicol palmitate has good oral bioavailability while the stable form A does not and as a consequence the limit of form A permissible in a product is strictly controlled 1 .

Numerous crystal engineering approaches to produce metastable forms are used pharmaceutically, including formation of salts, hydrates and solvates. These forms are of importance, not just for their physicochemical properties but also because they may affect ease of manufacture, purification or patentability. In the past decade the concept of pharmaceutical co-crystals has been introduced, defined as solid forms with unit cells comprising at least two compounds both of which interact by hydrogen bonding and/or other non-covalent bonds both of which are ordinarily solids at room temperature and pressure ${ }^{2}$. Co-crystals open the intriguing formulation strategy of creating crystal forms with improved physicochemical properties and acceptable stability. The co-former may be a stabilising excipient or a second active drug. For example, co-crystals of indomethacin-saccharin ${ }^{3}$, norfloxacin-isonicotinamide ${ }^{4}$ or carbamazepine with various compounds ${ }^{5-8}$ show improved solubility while piracetamL-tartaric acid co-crystals show better hygroscopicity ${ }^{9}$. 
Understanding whether a particular active drug can be formulated as a co-crystal is therefore commercially important. Screening for physical forms typically commences by growing crystals from solvents of different polarities, but this can be laborious and time consuming. For example, Seaton et al ${ }^{10}$ grew 2:1 co-crystals of benzoic acid (BA) and isonicotinamide (IsoNCT) from aqueous solution over 1 month. Such time scales are not particularly convenient for early development phase screening, when a rapid assessment of likely co-crystal formation is needed. Other methods, such as drop grinding ${ }^{11}$ or computational screening ${ }^{12}$, may be used but new methods for cocrystal screening, especially if they are quick and utilise small amounts of material, will always be of value.

In principle, any method that can break the crystal lattice bonds of the co-forming crystals and then allow mixing and reformation of bonds can be used to screen for co-crystals. We have shown previously how ink-jet printing small volumes of solutions can be used to prepare co-crystals, with the fast evaporation rates directing rapid crystallization often to metastable forms ${ }^{13}$. However, this approach requires the use of suitable solvents in which the co-formers have appreciable solubility and are suitable for printing. A solvent-free method would therefore be attractive.

Since rapid heating above the melt followed by quench cooling should facilitate molecular rearrangement, we wondered whether irradiating a dry powder blend of co-crystal formers with a high-power source, in this case a carbon dioxide $\left(\mathrm{CO}_{2}\right)$ laser, might be an effective approach. $\mathrm{CO}_{2}$ lasers have many applications in the medical (tissue ablation) ${ }^{14}$ and chemical (fabrication of microfluidic arrays) ${ }^{15}$ but we are not aware of any studies reporting their use for changing the crystalline form of a solid sample. Hence, the specific aim of this work was to determine whether $\mathrm{CO}_{2}$ laser irradiation of a powder blend facilitated co-crystal formation. We tested the 
hypothesis with a training set of known co-crystal formers and report the effect of irradiation parameters on the success and/or extent of conversion.

\section{Materials and methods}

Caffeine (CAF), oxalic acid (OXA), malonic acid (MALO), water (HPLC grade), methanol, ethanol and chloroform were purchased from Sigma-Aldrich (UK) and used as received.

Preparation of co-crystals by $\mathrm{CO}_{2}$ laser irradiation

A 40W CO 2 laser (Full Spectrum Laser LLC, Las Vegas, US) was used for this study. An image of a square $(3 \mathrm{~cm} \times 3 \mathrm{~cm}, 300 \mathrm{dpi})$ was used as a template for irradiation experiments. Binary dry powder blends of co-formers (respective masses CAF, 19.41 mg, OXA, $9.0 \mathrm{mg}$, MALO, $10.41 \mathrm{mg}$ ), prepared by physically shaking and tumbling the powders in a sample vial for $5 \mathrm{~min}$, were spread as a thin layer over aluminium foil (covering an area of the $3 \mathrm{~cm} \times 3 \mathrm{~cm}$ ). The focal length of the laser was $6.8 \mathrm{~cm}$. The laser allows user variation of power $(\mathrm{P})$ and raster speed $(\mathrm{S})$; various combinations were used (S60 and S50; P50 and P60; the number reflects the percentage of the maximum speed or power that the laser could achieve). Irradiated samples were removed from the foil to a small vial and stored in a desiccator over phosphorous pentoxide at ambient temperature until further analysis.

Preparation of reference co-crystals

All reference co-crystals were prepared by slow evaporation according to literature methods. CAF:OXA was prepared by dissolving CAF $(4.85 \mathrm{~g})$ and OXA $(1.12 \mathrm{~g})$ in 7:2 MeOH: $\mathrm{CHCl}_{3}(90 \mathrm{~mL})$. CAF:MALO was prepared by dissolving CAF (1.43 g) and 
MALO $(0.38 \mathrm{~g})$ in $30: 1 \mathrm{MeOH}: \mathrm{CHCl}_{3}(30 \mathrm{~mL})$. Solutions were kept at room temperature to allow the slow evaporation and precipitation. When the crystals had formed, they were isolated by filtration and stored in a desiccator over phosphorous pentoxide at ambient temperature until further analysis.

\section{X-Ray Powder Diffraction (XRPD)}

Data were collected on a Miniflex 600 diffractometer (Rigaku, Tokyo, Japan) with $\mathrm{Cu}$ Ka radiation at $40 \mathrm{kV}$ and $15 \mathrm{~mA}$ using a zero background holder. Scanning was performed from $5^{\circ}-35^{\circ} 2 \theta$ at $0.01^{\circ} 2 \theta$ step size and speed $5^{\circ} 2 \theta / \mathrm{min}$.

Differential Scanning Calorimetry (DSC)

DSC measurements were made with a Q2000 (TA Instruments, LLC, USA). Samples (3-5 mg) were encapsulated in Tzero aluminium pans and lids. The heating rate was $100{ }^{\circ} \mathrm{C} / \mathrm{min}$ and a nitrogen purge $(50 \mathrm{~mL} / \mathrm{min})$ was used. The instrument was calibrated using a standard reference material (indium, $T_{\mathrm{m}}=156.6, \Delta H=28.71 \mathrm{~J} / \mathrm{g}$ ) in accordance with the manufacturer's instructions. Data were analysed with Universal Analysis 2000 (TA Instruments, LLC, USA). Experiments were performed in triplicate, and the crystallization and melting values were reported as extrapolated onset ( $\left.T_{\text {onset }}\right)$.

Fourier-Transform Infrared (FT-IR)

Data were obtained with a 100 FT-IR spectrophotometer (Perkin Elmer). The spectrum of an empty cell was used as the background. The scan was performed in the range of 4000 to $650 \mathrm{~cm}^{-1}$ for each sample at ambient conditions. Spectrum Express software (version 2008) was used to process the data. 
Scanning Electron Microscopy (SEM)

Samples were mounted on an aluminium stage using adhesive tape and sputtercoated with gold (Quorum model Q150, Quorum Technology, UK) at 40 mA. Images were collected using an SEM (SEM, Quanta 200 FEG, FEI, Netherlands).

\section{Results and discussion}

The general principle by which laser irradiation might be expected to change physical form is that during irradiation a lot of energy is imparted to the sample, causing a rapid, but localised, rise in temperature. Because the laser is moved in a raster pattern across the sample, energy is imparted for only a short $(<1 \mathrm{~s})$ period of time, so there should be a relatively rapid fall in temperature back to that of the stage. Thus, crystalline particles melt, mix and are then forced to crystallise rapidly upon cooling. If a co-crystal solid form thermodynamically easier to form (i.e. if it is metastable with respect to either of the starting crystalline forms of the individual components) then co-crystals should result after irradiation. The balance of power (P) and raster speed (S) is important because it is necessary to impart to the sample enough energy for it to rise sufficiently in temperature to melt, but not so much that it degrades. It is also necessary to ensure the powder blends are thoroughly dry-mixed prior to irradiation and that they are spread thinly, so that all the material is heated sufficiently to melt.

The CAF:OXA system represents a non-congruently soluble co-crystal pair that can be quite challenging to co-crystallise. Trask et $a l^{16}$, successfully prepared 2:1 CAF:OXA by co-grinding and slow solvent evaporation while Daurio et al ${ }^{17}$ used hotmelt extrusion. XRPD patterns for the 2:1 CAF:OXA system are shown in Figure 1. It is apparent that the patterns for the irradiated samples are different from those of the 
starting materials or the physical blend, and match the pattern of the reference cocrystal. In particular, the characteristic intensity maxima at $11.2^{\circ}$ and $22.3^{\circ}$ seen for CAF and OXA starting materials respectively are absent from the co-crystal and irradiated patterns, while intensity maxima at 11.3, 12.0, 15.7, 17.1, 18.1, 21.8, 24.2, $26.6,27.7,28.2,29.9,30.8$ and $\left.32.0^{\circ}\right)$, characteristic of the co-crystal and in good agreement with the literature ${ }^{17-21}$, are seen in both irradiated samples. In all cases, irradiation with settings of P50:S50 produced slightly better co-crystals than irradiation at P60:S60. Irradiating at higher powers tended to degrade samples and irradiating at lower powers did not result in clear formation of co-crystals (data not shown).

Further characterisation of the physical form of the irradiated samples was performed with DSC and FTIR. Note here that we opted to use fast heating rates in the DSC because these increase the signal-to-noise ratio of the data while also minimising the time the sample is exposed to high temperatures (which is particularly important when studying metastable physical forms ${ }^{22}$ ). The DSC data, Figure 2, clearly show that the melting point of the reference co-crystal and irradiated samples, $210^{\circ} \mathrm{C}$, was different from that of the pure compounds and physical mixture. Melting of the cocrystal at $210^{\circ} \mathrm{C}$ is in good agreement with literature ${ }^{17,20}$. Pure CAF sublimes at 178 ${ }^{\circ} \mathrm{C}$ and melts at $236^{\circ} \mathrm{C}$ (in an open pan, melting would not be observed) while pure OXA shows numerous endothermic transitions, again ascribed to sublimation/melting. The absence of any sublimation peaks from the irradiated samples is good evidence that the co-crystal has formed. The physical mixture also shows evidence of the co-crystal melt, presumably because the co-crystal itself has formed during heating in the DSC. This observation substantiates the idea that physical form change during irradiation with the laser occurs because of a rapid rise in temperature. The sample irradiated at settings of P60:S60 showed characteristics of the powder blend, in that the melting endotherm at ca. $210^{\circ} \mathrm{C}$ was a doublet and 
an additional endotherm was seen at ca. $160^{\circ} \mathrm{C}$. In agreement with the XRPD data discussed above, this indicates that irradiating the sample at the higher power probably caused some degradation of the co-formers.

FT-IR spectra, Figure 3, confirm the irradiated samples have the same absorbance bands as the reference co-crystal. The $\mathrm{C}=\mathrm{O}$ bending and stretching bands shifted from $700,743,1646$ and $1695 \mathrm{~cm}^{-1}$ for CAF to $706,748,1659$ and $1700 \mathrm{~cm}^{-1}$ for the co-crystal and irradiated samples, while $\mathrm{C}-\mathrm{H}$ bending bands shifted from 862 and $1239 \mathrm{~cm}^{-1}$ to 899 and $1228 \mathrm{~cm}^{-1}$. In addition, a change in hydrogen bonding was observed in the $\mathrm{C}-\mathrm{H}$ stretching band, which shifted from $3112 \mathrm{~cm}^{-1}$ in the pure material to $3121 \mathrm{~cm}^{-1}$ in the co-crystal and irradiated samples ${ }^{19,21,23-24}$. On the other hand, the IR spectrum of the physical mixture matched those of CAF and OXA when superimposed, indicating there was no interaction between CAF and OXA after mixing.

SEM images (Figure 4) reveal differences in morphology and size between the starting materials, reference co-crystals and irradiated samples. Pure CAF was clumped with needle-shaped crystals, while OXA was seen as large plates. All the irradiated samples showed clusters of needle-like crystals, similar to the reference co-crystals. One difference between the irradiated samples and the reference cocrystal was the particle size, the irradiated samples containing smaller crystals. The reference prepared from solvent crystallization was found to be large and plate-like (ca. $50 \mu \mathrm{m}$ ), which is in good agreement with previous studies ${ }^{18-19}$. Chung and $\mathrm{Kim}^{19}$ noted nano co-crystals could be obtained by adding polymers, while laser irradiation produced crystals of ca. $5 \mu \mathrm{m}$. The small size is probably because molecular rearrangement and crystal growth can only occur while the material has sufficient energy following irradiation, and this is likely to be for a short time. One potential application of this approach, therefore, is preparation of particles for pulmonary 
delivery, where an average particle size below $5 \mu \mathrm{m}$ is required for successful delivery to the lung.

Formation of the 2:1 CAF:MALO co-crystal has been reported by solvent evaporation and solid-state grinding ${ }^{16,21}$. The XRPD patterns recorded here, Figure 5, show that the irradiated samples had the same characteristic set of intensity maxima $(7.6,11.0$, $12.2,15.6,17.3,17.7,18.7,20.3,21.9,22.8,23.2,24.6,27.2,28.4,30.6$ and $\left.31.4^{\circ}\right)$ as the reference co-crystal, while the characteristic maxima for CAF and MALO $\left(11.2^{\circ}\right.$ and $23.2^{\circ}$, respectively) were absent. The patterns are consistent with those reported by Nishijo et $\mathrm{al}^{21}$.

The DSC data, Figure 6, show melting of the co-crystal at ca. $132^{\circ} \mathrm{C}$, with a higher temperature endotherm at ca. $231^{\circ} \mathrm{C}$, most likely associated with possible recrystallisation and sublimation of CAF. The thermal behaviour of the irradiated samples matches that of the co-crystal. The thermal trace of the physical mixture is complex, showing a broad endotherm commencing at ca. $100^{\circ} \mathrm{C}$. Since this corresponds to the melting point of MALO, it may be the case that the data reflect melting of MALO, possible dissolution and/or recrystallization with CAF and then sublimation of CAF at higher temperatues. It is clear, however, that the co-crystal was not present initially in powder blend.

The FTIR spectra, Figure 7, confirm the irradiated samples have similar bonding to the reference co-crystal. The bands associated with $\mathrm{C}=\mathrm{O}$ bending and stretching shift from $700,743,744,1646,1645$ and $1695 \mathrm{~cm}^{-1}$ for CAF to $706,748,1659,1657$ and $1700 \mathrm{~cm}^{-1}$ for the 2:1 CAF:MALO co-crystal and irradiated samples, while the absorption bands of the $\mathrm{C}-\mathrm{H}$ bending shift from 862 and $1239 \mathrm{~cm}^{-1}$ to 902 and 1228 $\mathrm{cm}^{-1}$. Changes in hydrogen bonding are seen in the $\mathrm{C}-\mathrm{H}$ stretching vibration, which shifts from 3112 and $3109 \mathrm{~cm}^{-1}$ to 3121 and $3122 \mathrm{~cm}^{-1}$ while new interactions in the 
co-crystal are indicated by $\mathrm{N}-\mathrm{H}$ stretching, $\mathrm{C}=\mathrm{O}$ and $\mathrm{C}-\mathrm{H}$ vibrations at 3122,1698 , 1658 and $1611 \mathrm{~cm}^{-1}$. The spectrum of the physical mixture matched those of CAF and MALO when superimposed, which indicated that there was no interaction between CAF and MALO after mixing.

Finally, visual confirmation that a change in morphology occurs upon irradiation is provided by SEM images, Figure 8. While the larger crystals of MALO are not seen in the irradiated samples, needle-like crystals are seen emerging from a homogeneous core.

Interestingly, although the results are not shown here, we were only able to make cocrystals with powder mixtures where at least one co-former showed significant sublimation (both caffeine and malonic acid sublime to an appreciable extent, while oxalic acid shows a small extent of sublimation). This suggests that the mechanism is more complex than just a simple rise in temperature melting the material as the laser moves across the powder bed. Dhumal et $\mathrm{al}^{25}$ suggested a mechanism to explain co-crystallization by solid-state grinding involving mass transfer via vapour diffusion. In this model, nuclei form in the highly saturated and stoichiometric vapour above the molten sample. Vapourised material then re-condenses to form crystals on a substrate where co-crystal growth occurs. It seems likely that a mechanism of this sort, involving interaction of compounds in the gaseous state, is involved with laser irradiation and this explains when the whole sample can convert to a new crystal form, even though it is the surface of the powder that is exposed to irradiation energy. 


\section{Conclusion}

$\mathrm{CO}_{2}$ laser irradiation has been demonstrated as a novel, rapid and solvent-free method for the preparation of pharmaceutical co-crystals. Co-crystals of 2:1 CAF:OXA and 2:1 CAF:MALO were successfully produced. Various parameters, such as laser speed and laser power, were found to be key factors in producing high quality co-crystals. Co-crystals were only successfully produced from co-formers that were sublimable, indicating that nucleation in the vapour phase was the probably mechanism of formation. 


\section{References}

(1) Aguiar, A.J.; Krc, J.; Kinkel, A.W.; Samyn, J.C. J. Pharm. Sci. 1967, 56, 847-853.

(2) Almarsson, O.; Zaworotko, M.J. Chem. Commun. 2004, 17, 1889-1896.

(3) Basavoju, S.; Bostrm, D.; Velaga, S. Pharm. Res. 2008, 25, 530-541.

(4) Basavoju, S.; Bostrm, D.; Velaga S. Cryst. Growth Des. 2006, 6, 2699-2708.

(5) Hickey, M.; Peterson, M.; Scoppettuolo, L.; Morrisette, S.; Vetter, A.; Guzmán, H.; Remenar, J.F.; Zhang, Z.; Tawa, M.D.; Haley, S.; Zaworotko, M.J.; Almarsson, O. Eur. J. Pharm. Biopharm. 2007, 67, 112-119.

(6) Nehm, S.; Rodríguez-Spong, B.; Rodríguez-Hornedo, N. Cryst. Growth Des. $2006,6,592-600$.

(7) Rodríguez-Hornedo, N.; Nehm, S.; Seefeldt, K.; Pagán-Torres, Y.; Falkiewicz, C. Mol. Pharm. 2006, 3, 362-367.

(8) Porter, W.; Elie, S.; Matzger, A. Cryst. Growth Des. 2008, 8, 14-16.

(9) Viertelhaus, M.; Hilfiker R.; Blatter, F.; Neuburger, M. Cryst. Growth Des. 2009, 9, 2220-2228.

(10) Seaton, C.C.; Parkin, A.; Wilson, C.C.; Blagden, N. Cryst. Growth Des., 2009, 9, 47-56.

(11) Patel, J.R.; Carlton, R.A.; Needham, T.E.; Chischester, C.O.; Vogt, F.G. Int J Pharm., 2012, 436, 685-706.

(12) Musumeci, D.; Hunter, C.A.; Prohens, R.; Scuderi, S.; McCabe, J.F. Chem Sci., 2011, 2, 883-890.

(13) Buanz, A.B.M.; Telford, R.; Scowen, I.J.; Gaisford, S. CrystEngComm. 2013, 15, 1031-1035.

(14) Landthaler, M.; Haina, D.; Hohenleutner, U.; Seipp, W.; Waidelich, W.; Braunfalco, O. Hautarzt, 1988, 39, 198-204.

(15) Prakash, S.; Kumar, S. Int J Pres Eng Manufac., 2015, 16, 361-366.

(16) Trask, A.V.; Motherwell, W.D.S.; Jones, W. Cryst. Growth Des., 2005, 5, 10131021. 
(17) Daurio, D.; Medina, C.; Saw, R.; Nagapudi, K; Alvarez-Núñez, F.

Pharmaceutics, 2011, 3, 582-600.

(18) Aher, S.; Dhumal, R.; Mahadik, K.; Ketolainen, J.; Paradkar, A. Pharm. Dev. Tech., 2013, 18, 55-60.

(19) Chung, J.; Kim, I.W. J. Cryst. Growth, 2011, 335, 106-109.

(20) Medina, C.; Daurio, D.; Nagapudi, K.; Alvarez-Nunez, F. J. Pharm. Sci., 2010, 99, 1693-1696.

(21) Nishijo, J.; Takenaka, F.; Tanaka, I. Yakugaku Zasshi, 1980, 100, 732-738.

(22) Gaisford, S.; Buanz, A.B.M.; Jethwa, N. J Pharm Biomed Anal., 2010, 53, 366370.

(23) Joseph, J.; Jemmis, E.D. J. Am. Chem. Soc., 2007, 129, 4620-4632

(24) Nolasco, M. M.; Amado, A.M.; Ribeiro-Claro, P.J.A. ChemPhysChem, 2006, 7, 2150-2161.

(25) Dhumal, R.; Kelly, A.; York, P.; Coates, P.; Paradkar, A. Pharm. Res., 2010, 27, 2725-2733. 


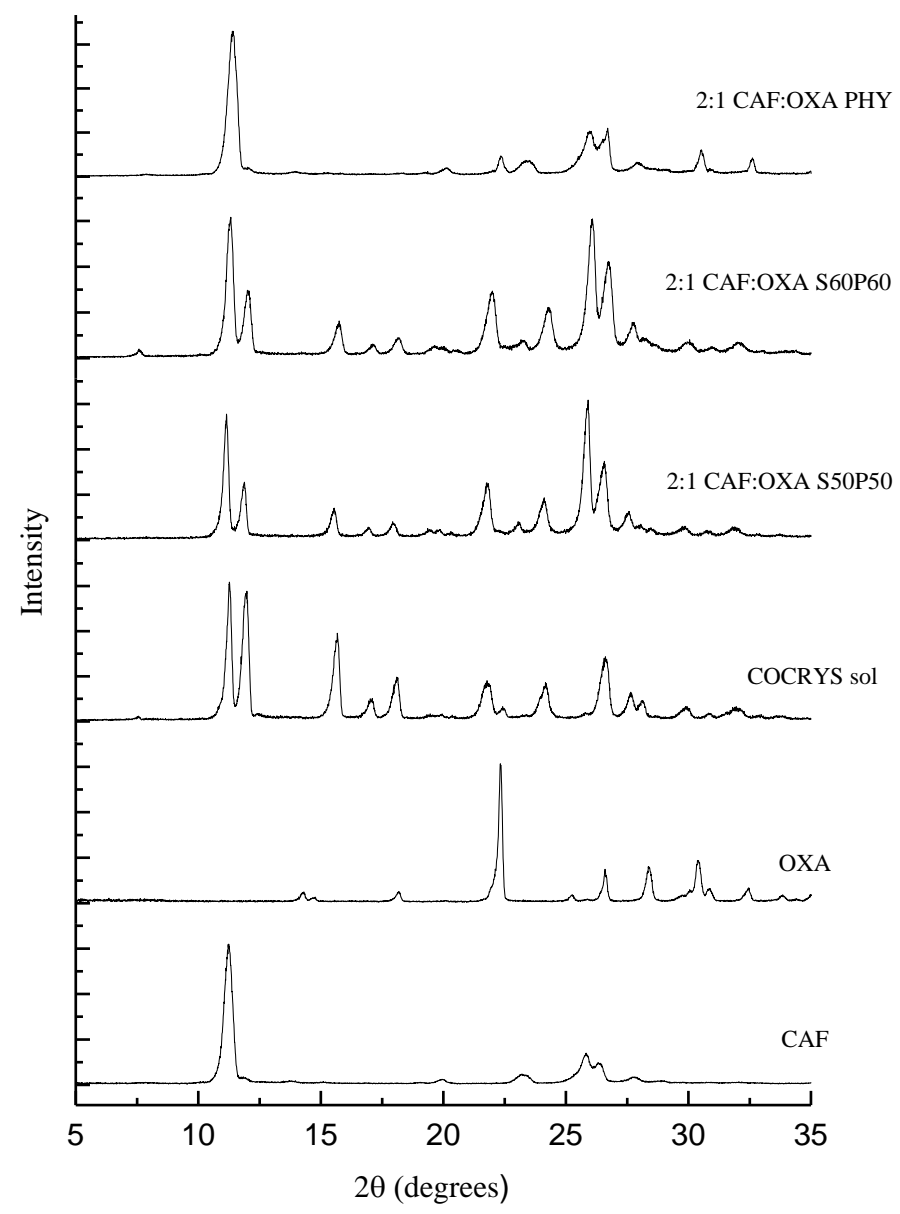

Figure 1: X-ray powder diffraction patterns for caffeine and oxalic acid, showing reference patterns for the crystalline starting materials, a physical mixture (PHY), a reference co-crystal prepared from solution (sol) and samples irradiated at various $P$ and $S$ settings. 


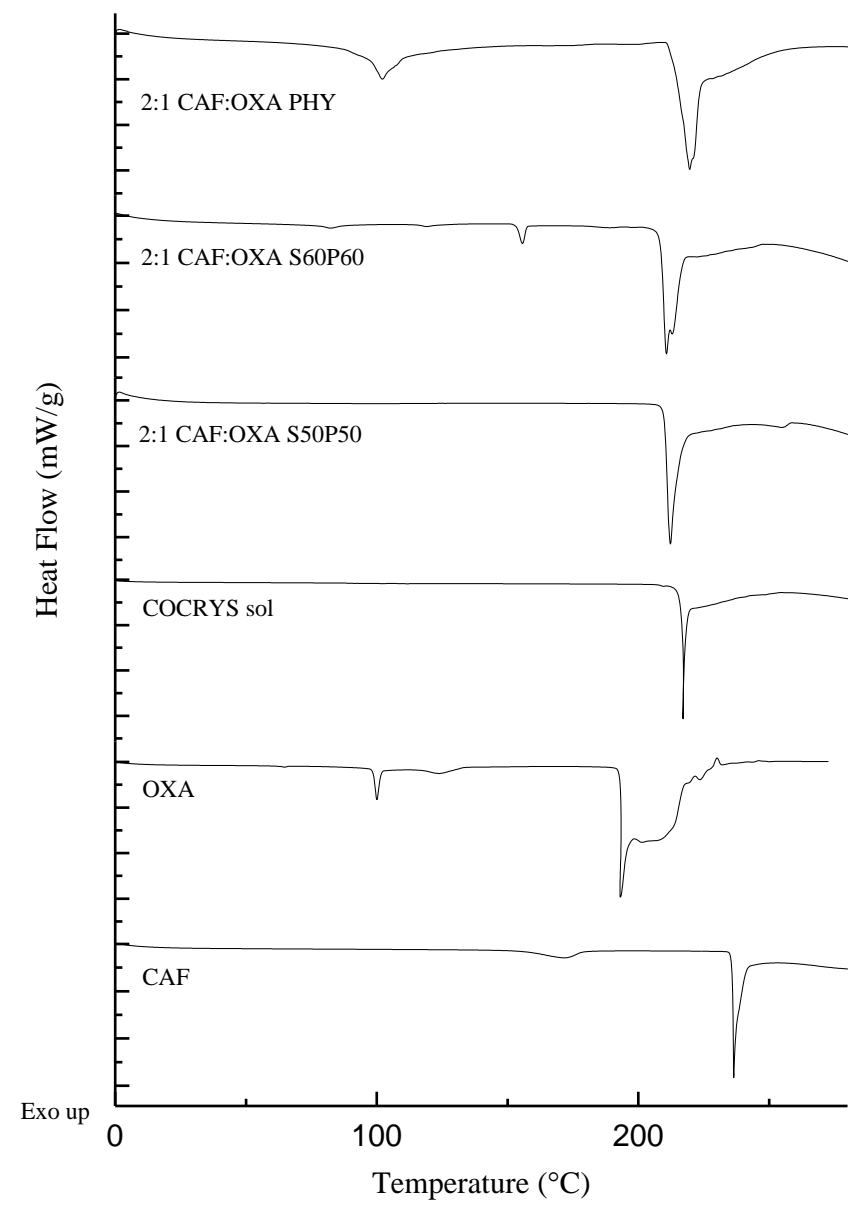

Figure 2. DSC thermal traces for CAF and OXA, a physical mixture (PHY), a reference co-crystal prepared from solution (sol) and samples irradiated at various $P$ and $S$ settings. 


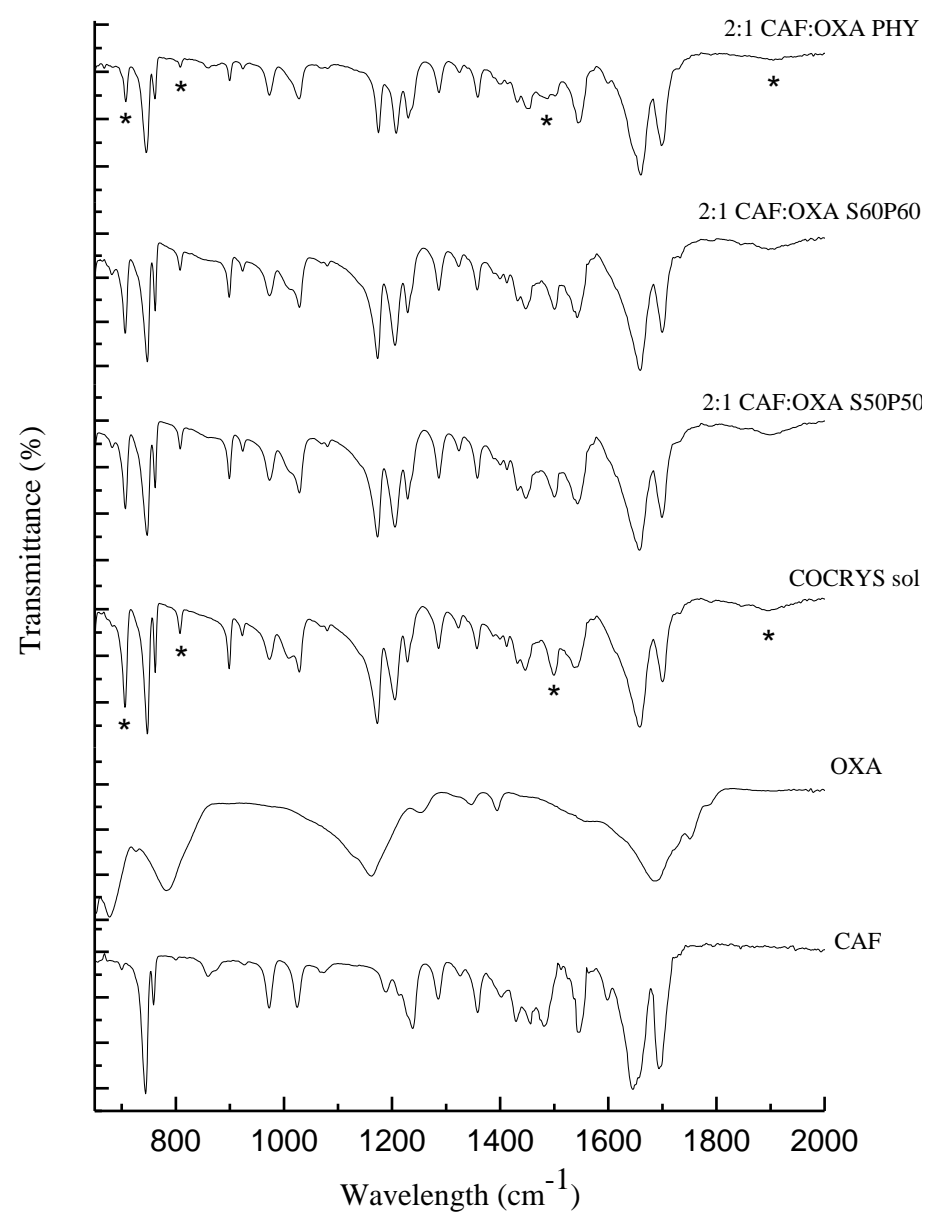

Figure 3. FTIR spectra for CAF and OXA, a physical mixture (PHY), a reference co-crystal prepared from solution (sol) and samples irradiated at various $\mathbf{P}$ and S settings. 


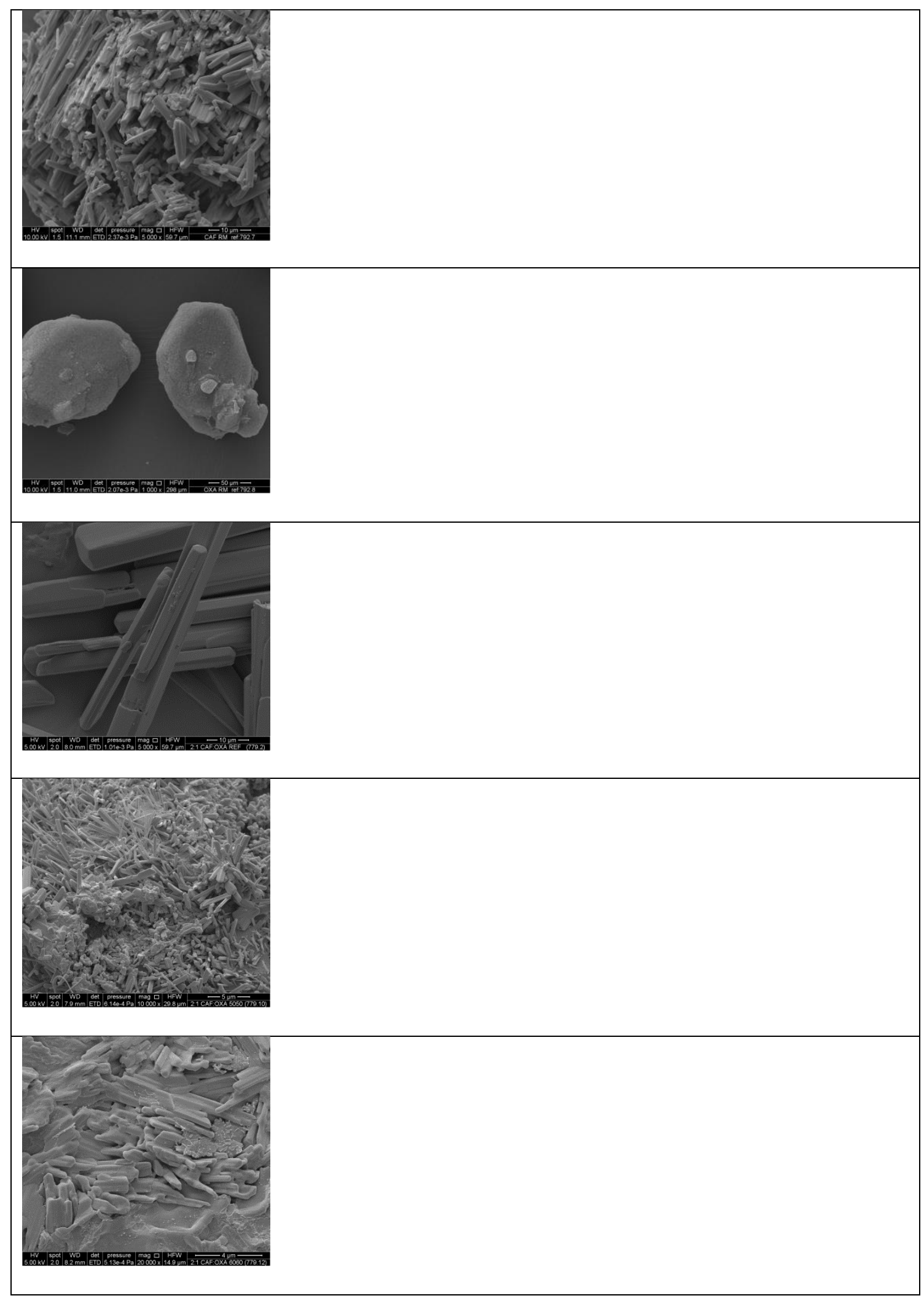

Figure 4. SEM images of (from top to bottom) CAF, OXA, the reference cocrystal, S50:P50 and S60:P60. 


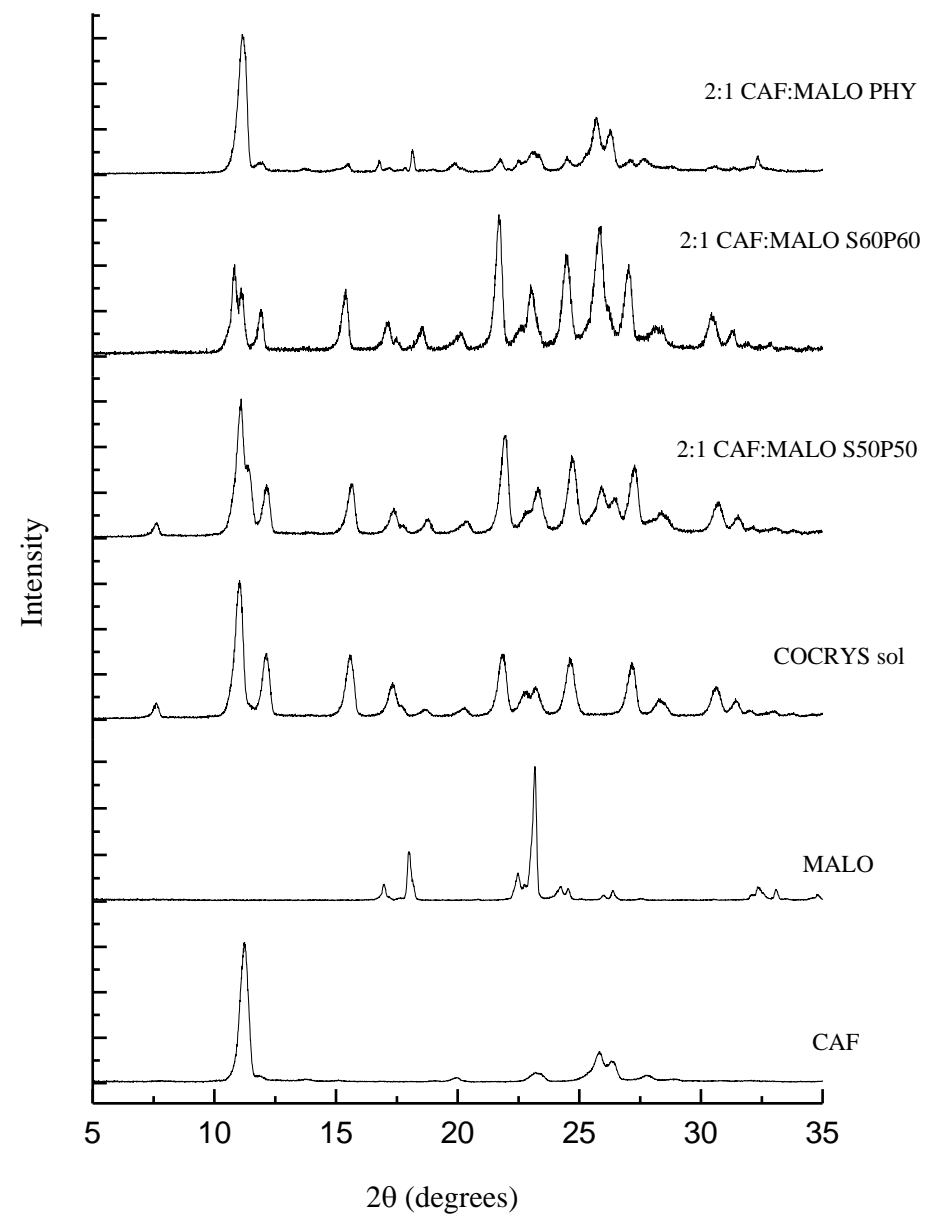

Figure 5: X-ray powder diffraction patterns for caffeine and malonic acid, showing reference patterns for the crystalline starting materials, a physical mixture (PHY), a reference co-crystal prepared from solution (sol) and samples irradiated at various $P$ and $S$ settings. 


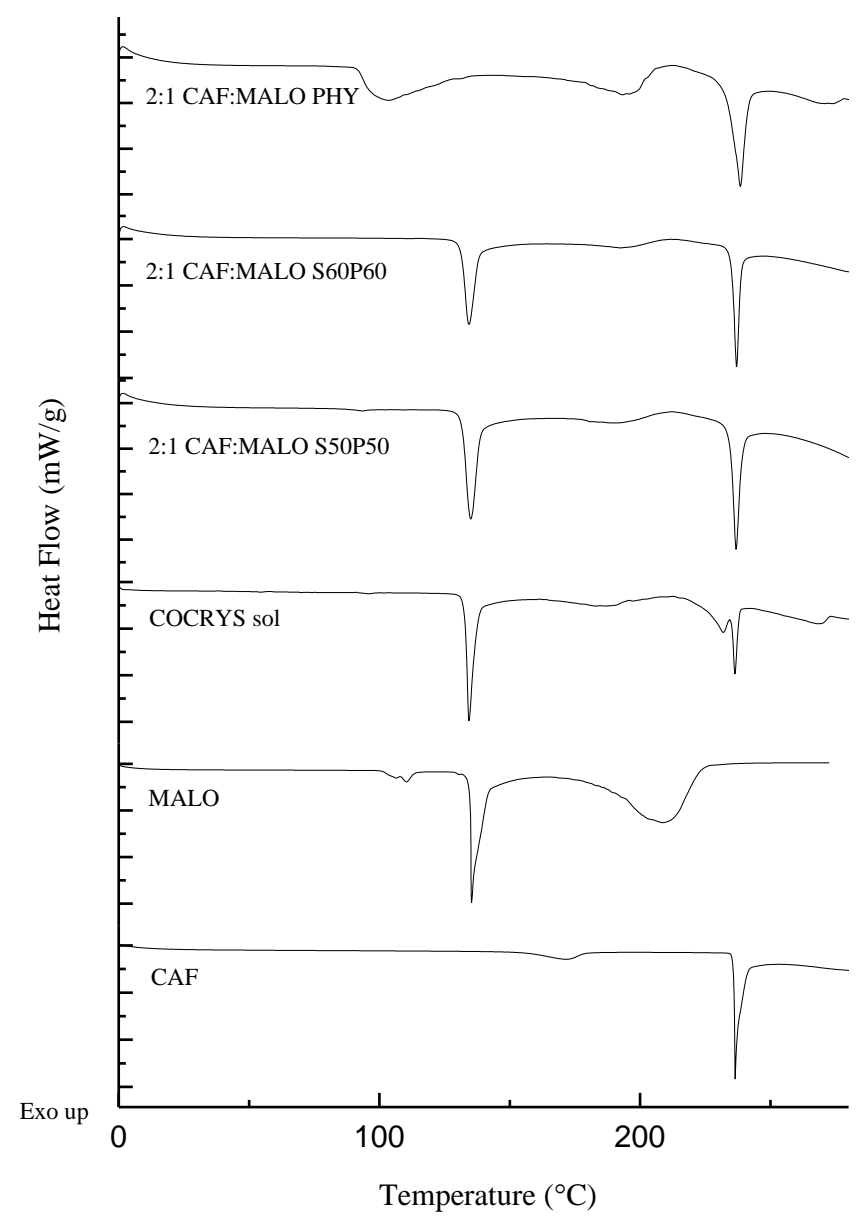

Figure 6. DSC thermal traces for CAF and MALO, a physical mixture (PHY), a reference co-crystal prepared from solution (sol) and samples irradiated at various $P$ and $S$ settings 


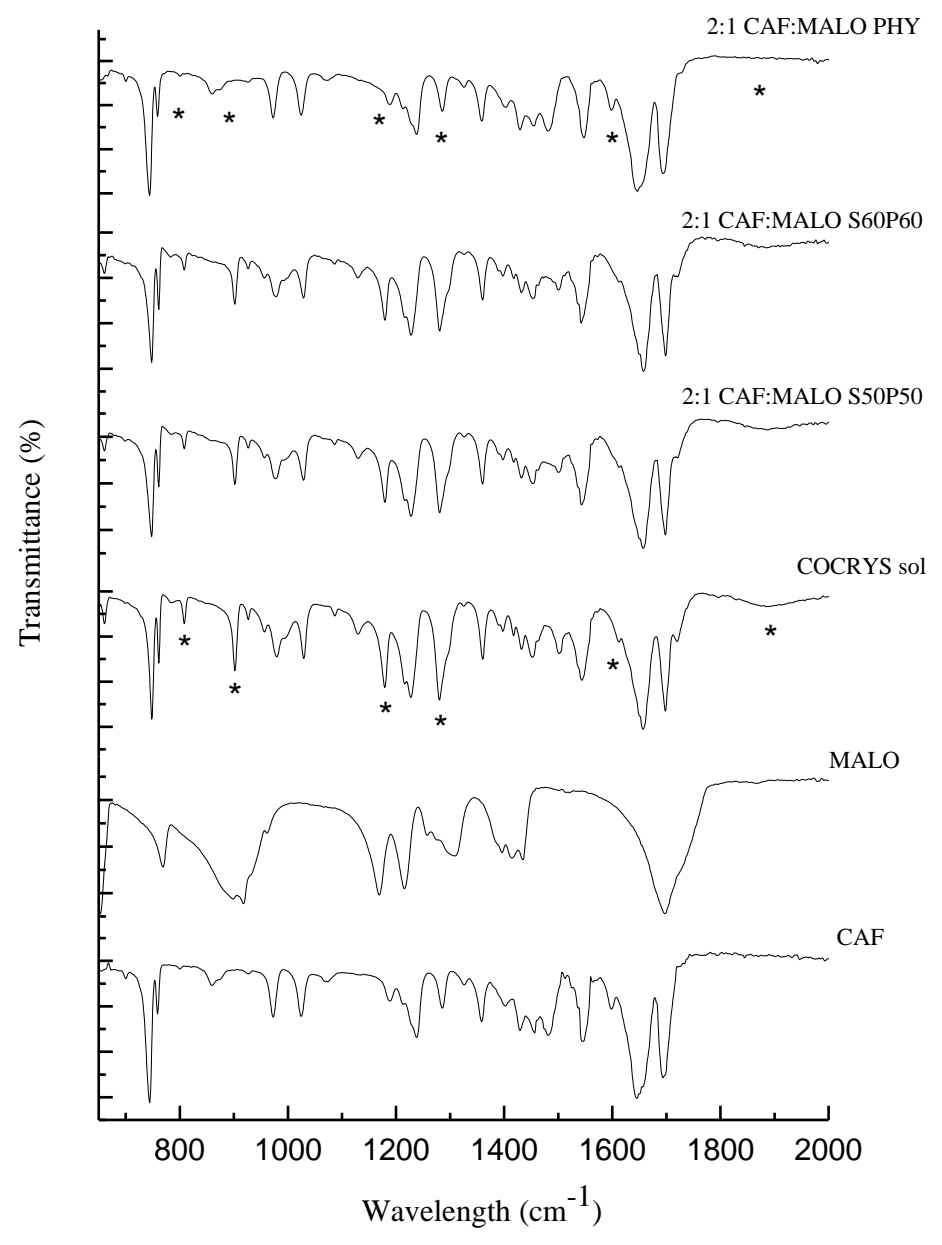

Figure 7. FTIR spectra for CAF and MALO, a physical mixture (PHY), a reference co-crystal prepared from solution (sol) and samples irradiated at various $P$ and $S$ settings 


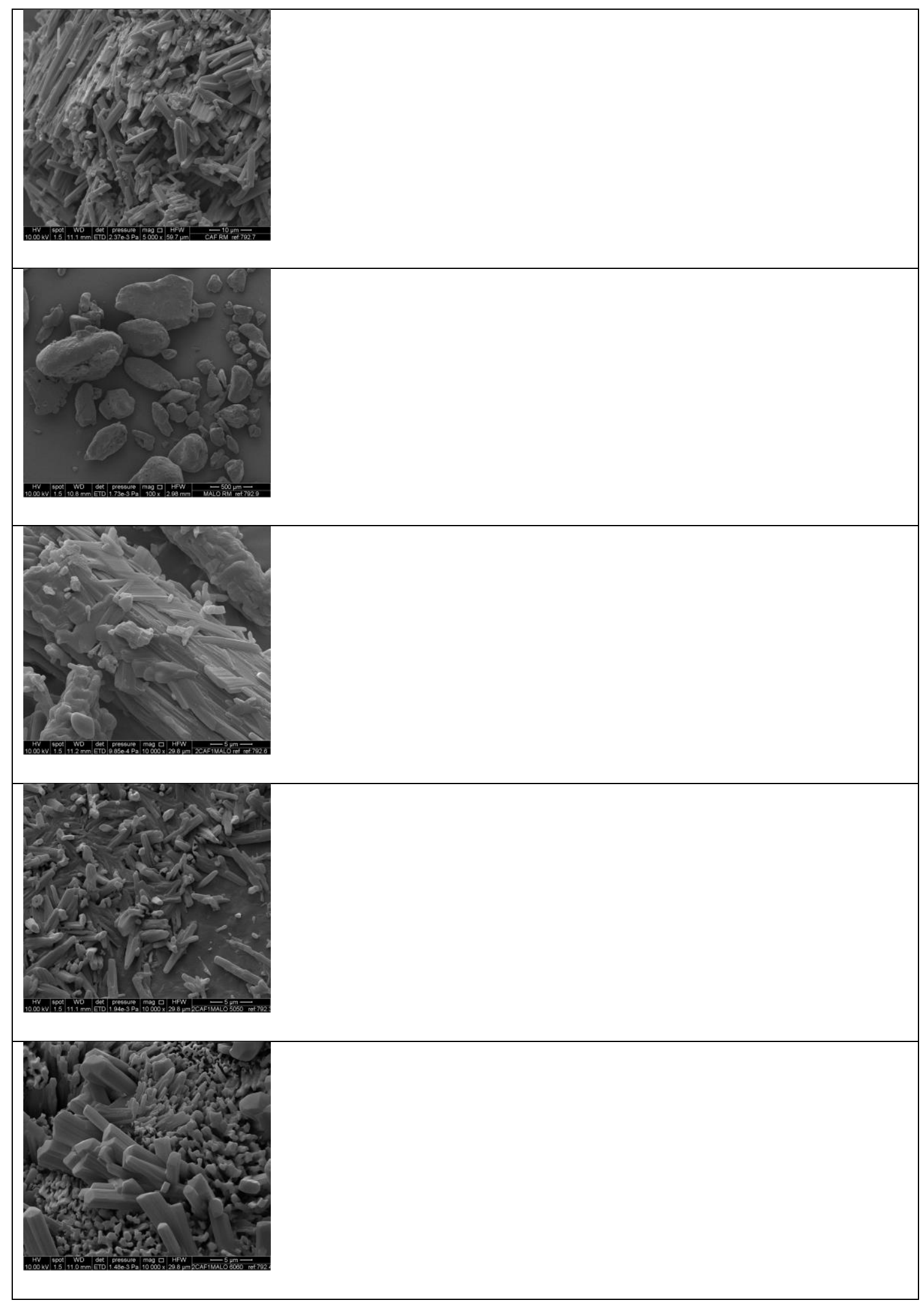

Figure 8. SEM images of (from top to bottom) CAF, MAL, the reference cocrystal, S50:P50 and S60:P60. 
For Table of Contents use only:

Manuscript title: A New Method for Producing Pharmaceutical Co-crystals: Laser Irradiation of Powder Blends

Author list: Varin Titapiwatanakun, Abdul W. Basit, Simon Gaisford

TOC Graphic:
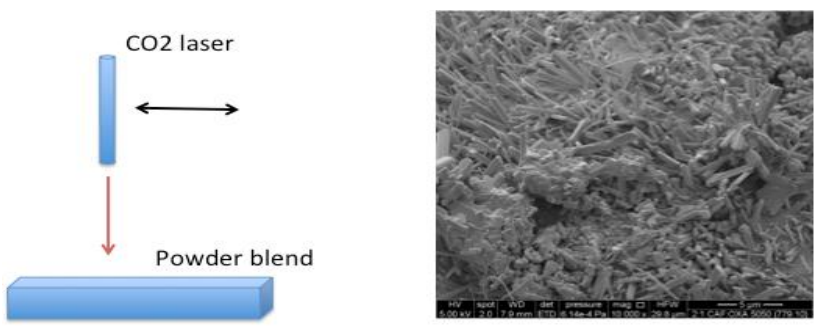

Synopsis: A high-power $\mathrm{CO}_{2}$ laser was used to irradiate powder blends of co-crystal formers, with the specific aim of trying to cause recrystallization to a co-crystal structure. It was found that sufficient thermal energy could be imparted to the powder to cause molecular rearrangement but that for co-crystals to form successfully, the co-formers needed to sublime to an appreciable extent. 